Series A

I. MATHEMATICA

597

\title{
MINIMALE SEPARIERTE \\ VEKTORRAUMLIMITIERUNGEN UND DER \\ GRAPHENSATZ
}

VON

STEN BJON

HELSINKI 1975

S U O M A L A I N N T I D E AKA TEMIA

doi:10.5186/aasfm.1975.597 
Copyright (C) 1975 by Academia Scientiarum Fennica ISSN 0066-1953

ISBN 951-41-0213.4

Vorgelegt am 18. Juni 1974

KESKUSKIRJAPAINO IIELSINKI 1975 


\section{Einleitung}

Wir nennen ein Objekt $(E, A)$ in einer Kategorie $\mathcal{K}$ separierter Limesvektorräume $(m)$-Objekt in $\mathcal{K}$, falls jeder Bimorphismus $(E, A) \rightarrow\left(F, \Lambda^{\prime}\right)$ nach einem Objekt $\left(F, A^{\prime}\right)$ in $\mathcal{K}$ ein Isomorphismus ist. Die Existenz nicht-trivialer $(m)$-Objekte wird in verschiedenen Kategorien von Limesvektorräumen mit Hilfe des Zorn'schen Lemmas nachgewiesen. Für lineare Abbildungen von einem Objekt in $\mathcal{K}$ nach einem $(m)$-Objekt in $\mathcal{K}$ wird einen Graphensatz bewiesen. Unter den betrachteten Kategorien ist die Kategorie $7 \overline{\bar{Q}} \dagger$ der sog. "stark ausgeglichenen» Limesvektorräume, die auch einer Abzählbarkeitsbedingung $(\dagger)$ genügen, die wichtigste. Die Kategorie der metrisierbaren, topologischen Vektorräume ist eine Unterkategorie von $\mathcal{V}^{\bar{C}} \dagger$ und die vollständigen, metrisierbaren, topologischen Vektorräume sind sogar $(m)$-Objekte in der vollen Unterkategorie der vollständigen, regulären Objekte in $\mathcal{V}^{\bar{C}}{ }^{\dagger}$. Die Limesstruktur auf einem Objekt in $\overline{\bar{F}} \dagger$ kann durch eine Menge translationsinvarianter Metriken definiert werden, und umgekehrt definiert eine Menge $\Delta$ translationsinvarianter Metriken auf einem Vektorraum $E$ eine Limitierung $\Lambda$ auf $E$ derart, dass $(E, \Lambda)$ Objekt in $\mathcal{V} \bar{E} \dagger^{-}$ist, falls $\Delta$ und die Elemente in $\Delta$ gewisse Eigenschaften haben. Im letzten Abschnitt wird die Vollständigkeit gewisser absolutkonvexer Räume durch ihre Einbettungseigenschaften charakterisiert.

Begriffe und Bezeichnungen. Mit $|\mathcal{X}|$ bezeichnen wir die Objektklasse einer Kategorie $\mathcal{K}$. Unter Vektorraum verstehen wir einen Vektorraum über dem Körper $\mathbf{K}$ der reellen oder komplexen Zahlen. $\boldsymbol{V}$ sei der Nullumgebungsfilter bezüglich der Betragtopologie auf K. Ein Filter $\mathcal{F}$ auf einem Vektorraum $E$ heisst nach [6] ausgeglichen (equable), falls gilt $\boldsymbol{V} \cdot \mathcal{F}=\mathcal{F}$. Wir sagen, dass $\mathcal{F}$ stark ausgeglichen ist, falls diesüber gilt $\mathcal{F}+\mathcal{F}=\mathcal{F}$. Eine Vektorraumlimitierung $A$ auf $E$ und der Limesvektorraum $(E, \Lambda)$ heissen (stark) ausgeglichen, falls es für jedes $\mathcal{F} \in \Lambda 0$ ein (stark) ausgeglichenes $\mathscr{C}_{\mathcal{A}} \in \Lambda 0$ mit $\mathscr{C}_{1} \leq \mathcal{F}$ gibt. Die Vektorraumlimitierung $\Lambda$ und der $\operatorname{Raum}(E, \Lambda)$ heissen absolutkonvex, falls es zu jedem Filter $\mathcal{F} \in \Lambda 0$ einen Filter $\mathscr{G} \in \Lambda 0$ gröber als $\mathcal{F}$ mit einer Basis aus absolutkonvexen Mengen gibt.

Diese arbeit wurde von Svenska Kulturfonden unterstützt. 
Es sei $E$ ein Vektorraum und $\mathbf{R}^{+}$die Menge der nichtnegativen reellen Zahlen. Abbildungen $q: E \rightarrow \mathbf{R}+\cup\{\infty\}$ mit den Eigenschaften: (a) $q(0)=0$, (b) $q(\lambda x)=|\lambda| q(x), \lambda \neq 0, \lambda \in \mathbf{K}, x \in E$, (c) $q(x+y) \leq$ $q(x)+q(y), \quad x \in E, \quad y \in E$, nennen wir Pseudonormen. Auf der Menge $Q(E)$ der Pseudonormen auf $E$ sei $\leq$ die punktweise Ordnung. Eine Teilmenge $M$ von $Q(E)$ heisst saturiert, falls die Pseudonormen $q_{1}$ und $q_{2}$ genau dann in $M$ sind, wenn $\sup \left(q_{1}, q_{2}\right)$ in $M$ ist. Ein Filter auf $Q(E)$ heisst saturiert, falls er eine Basis aus saturierten Mengen besitzt.

Jede Pseudonorm $q \in Q(E)$ definiert eine absolutkonvexe Teilmenge $U_{q}=\{x \mid q(x) \leq 1\}$ von $E$ und jede Teilmenge $M \subset Q(E)$ definiert einen Filter $\mathcal{F}_{M}=\left[\left\{U_{q} \mid q \in M\right\}\right]$ auf $E$. Nach [2] definiert ein Filter $\psi$ auf $Q(E)$ durch $\Lambda 0=\left[\left\{\mathcal{F}_{M} \mid M \in \psi\right\}\right], \quad \Lambda x=x+\Lambda 0$ eine absolutkonvexe Limitierung $\Lambda$ auf $E$, falls

(I) $\lambda \psi \leq \psi$ für jedes $\lambda>0$

(II) für jedes $x \in E$ gibt es ein $M \in \psi$, so dass $q(x)<\infty$ für jedes $q \in M$ ist

erfüllt sind. Jede absolutkonvexe Limitierung kann in dieser Weise durch einen saturierten Filter auf $Q(E)$ mit den Eigenschaften (I) und (II) definiert werden. Falls $\Lambda$ ausgeglichen ist, können wir sogar voraussetzen, dass $\psi$ eine Basis aus saturierten Mengen $M$ mit $\lambda M=M$ für jedes $\lambda>0$ besitzt.

Für weitere Bezeichnungen wird auf [5] und [2] verwiesen.

\section{2. (m)-Objekte}

Es sei $V \mathscr{L}$ die Kategorie der separierten Limesvektorräume. Die Morphismen von $\mathfrak{V} \mathcal{L}$ seien die stetigen, linearen Abbildungen zwischen Limesvektorräumen. Neben $\mathscr{\mathscr { L }}$ betrachten wir folgende volle Unterkategorien von $\mathscr{V} \mathscr{L}$ :

$\mathcal{V}_{\mathscr{E}}=$ Kategorie der ausgeglichenen Limesvektorräume (vgl. [6]).

$\vee \bar{g}=$ " " stark ausgeglichenen Limesvektorräume.

Ve $="$ absolutkonvexen Limesvektorräume (vgl. [2]).

VEE $=$ " $\quad$ "ausgeglichenen, absolutkonvexen Limesvektorräume.

$\mathcal{V} M$ = Kategorie der Marinescu-Räume (vgl. [8]).

Wir bemerken, dass jede ausgeglichene, absolutkonvexe Limitierung stark ausgeglichen ist. Für einen ausgeglichenen Filter $\mathcal{F}$ mit absolutkonvexer Basis gilt nämlich: $\mathcal{F}+\mathcal{F}=\mathcal{F}$. 
Ist $\mathcal{K}$ eine Unterkategorie von $\mathcal{V} \mathscr{L}$, so bezeichnet $\mathcal{K}^{*}, \mathcal{K}^{\dagger}$ bzw. $\mathcal{K}^{l c}$ die volle Unterkategorie von $\mathscr{V} \mathscr{L}$, die genau alle Objekte $(E, \Lambda) \in|\mathcal{K}|$ mit der Eigenschaft $(*),(\dagger)$ bzw. $(l c)$ enthält.

(*) $(E, \Lambda)$ ist vollständig.

$(\dagger)$ Für jeden Filter $\mathcal{F} \in \Lambda 0$ gibt es einen Filter $C_{g} \in \Lambda 0$ mit $C_{g} \leq \mathcal{F}$, der eine abzählbare Filterbasis besitzt.

(lc) Der Raum $(E, A)$ ist lokalkompakt, d.h. jeder Filter $\mathcal{F} \in \Lambda 0$ enthält eine kompakte Menge (vgl. [11]).

Definition. Eine Limitierung $A$ auf einem Vektorraum $E$ heisse K-Limitierung auf $E$, wenn $(E, A) \in|\mathcal{K}|$ ist.

Definition. Ein Objekt $(E, A) \in|\mathcal{K}|$ heisse $(m)$-Objekt in $\mathcal{K}$, wenn jeder Bimorphismus $f:(E, A) \rightarrow\left(F, \Lambda^{\prime}\right)$ von $(E, \Lambda)$ nach einem Objekt $\left(F, A^{\prime}\right) \in|\mathcal{K}|$ ein Isomorphismus ist.

Offensichtlich ist $(E, \Lambda)$ genau dann ein $(m)$-Objekt in $\mathcal{K}$, wenn $\Lambda$ minimales Element in der geordneten Menge der $\mathcal{K}$-Limitierungen auf $E$ ist.

Beispiel 1. Jeder endlichdimensionale Raum in einer Unterkategorie $\mathcal{K}$ von $\vartheta \mathscr{L}$ ist $(m)$-Objekt in $\mathcal{K}$. Nach [10] ist nämlich die natürliche Topologie die einzige separierte Vektorraumlimitierung auf einem endlichdimensionalen Raum.

Beispiel 2. In der Kategorie der Banach-Räume ist jedes Objekt $(m)$ Objekt.

Satz 1. Es sei $\mathcal{K}$ gleich einer der Kategorien $V \mathscr{L}, V_{\mathscr{E}}, \mathcal{V E}_{\mathscr{E}}$, Ve, Vege oder VMn. Für jedes Objekt $(E, \Lambda) \in|\mathcal{K}|\left(\left|\mathcal{K}^{*}\right|,\left|\mathcal{K}^{\dagger}\right|\right.$, $\left|\mathcal{K}^{l c}\right|,\left|\mathcal{K}^{+*}\right|$ bzw. $\left.\left|\mathcal{X}^{+l c}\right|\right)$ gibt es einen Bimorphismus $(E, \Lambda) \rightarrow\left(F, \Lambda^{\prime}\right)$ nach einem $(m)$-Objekt $\left(F, \Lambda^{\prime}\right)$ in $\mathcal{K}\left(\mathcal{K}^{*}, \mathcal{K}^{\dagger}, \mathcal{K}^{l c}, \mathcal{K}^{+*}\right.$ bzw. $\left.\mathcal{K}^{+l c}\right)$.

K. Kutzler hat in [11] gezeigt, dass die feinste Vektorraumlimitierung auf einem Vektorraum eine lokalkompakte Marinescu-Limitierung ist. Da sie sich als induktiver Limes der natürlichen Topologien auf endlichdimensionalen Unterräumen darstellen lässt, erfüllt sie auch die Abzählbarkeitsbedingung ( $\dagger$ ). Die Kategorie $9 / 97+l c$ enthält also auch Räume, die algebraisch unendlichdimensional sind.

Beweis von Satz 1: Es sei $\left\{\Lambda_{\imath}, \iota \in I\right\}$ eine total geordnete Menge von $\mathcal{K}_{-}\left(\mathcal{K}^{*_{-}}, \mathcal{K}^{\dagger_{-}}, \mathcal{K}^{l_{-}}, \mathcal{K}^{\dagger *_{-}}\right.$bzw. $\left.\mathcal{K}^{+t c_{-}}\right)$Limitierungen auf $E$, die gröber als $\Lambda$ sind. Der Raum $\left(E, \Lambda_{1}\right)$ sei der induktive Limes (in der Kategorie der Limesräume) der Räume $\left(E, \Lambda_{\iota}\right), \iota \in I$, nach den Identitäten $i_{\varkappa}:\left(E, \Lambda_{\iota}\right) \rightarrow\left(E, \Lambda_{\varkappa}\right) \quad\left(\Lambda_{\iota} \geq \Lambda_{\varkappa}\right)$ auf $E$. Die Limitierung $\Lambda_{1}$ ist eine separierte Vektorraumlimitierung und es gilt: $\Lambda_{1} 0=\bigcup_{\iota \in I} \Lambda_{\iota} 0$. Aus dieser Gleichung folgt, dass die Limitierung $\Lambda_{1}$ mit den Limitierungen $\Lambda_{\imath}, \iota \in I$, ausgeglichen, stark ausgeglichen oder absolutkonvex ist und dass sie (*) 
oder $(\dagger)$ genügt, falls irgendeine oder beide dieser Beziehungen für alle $\Lambda_{\iota}$ gelten. Induktive Limites von Marinescu-Räumen bzw. lokalkompakten Limesräumen sind wieder Marinescu-Räume bzw. lokalkompakte Limesräume (siehe [8] bzw. [11]). Sind alle $\Lambda_{\iota}$ Marinescu- bzw. lokalkompakte Limitierungen, so gilt also dieses auch für $\Lambda_{1}$. Nach dem Zorn'schen Lemma gibt es eine minimale $\mathcal{K}_{-}\left(\mathcal{K}^{*_{-}}, \mathcal{K}_{-}^{t_{-}}, \mathcal{K}^{l_{-}}, \mathcal{K}^{+*_{-}}\right.$bzw. $\left.\mathcal{K}^{+c_{-}}\right)$ Limitierung $\Lambda^{\prime}$ auf $E$, die gröber als $\Lambda$ ist. Die Identität $(E, A) \rightarrow$ $\left(E, \Lambda^{\prime}\right)$ auf $E$ ist ein Bimorphismus nach dem $(m)$-Objekt $\left(E, \Lambda^{\prime}\right)$.

In Satz 1 brauchen wir die Kategorien $\mathcal{X} l c *$, wo $\mathcal{K}$ die in Satz 1 genannten Kategorien durchläuft, nicht in Betracht ziehen, denn nach Satz 2 ist $\mathcal{K}^{l c *}=\mathcal{K}^{l c}$.

Satz 2. Jeder lokalkompakte Limesvektorraum ist vollständig.

Beweis. Es sei $(E, \Lambda)$ ein lokalkompakter Limesvektorraum und $\mathscr{C}_{\mathcal{G}}$ ein Cauchy-Filter in $(E, \Lambda)$. Weiter sei der Filter $\mathcal{F} \in \Lambda 0$ so gewählt, dass $\mathscr{G}-\mathscr{G} \geq \mathcal{F}$ ist. Es gibt dann ein kompaktes $K \in \mathcal{F}$ und eine Menge $G \in \mathscr{G}$ derart, dass $G-G \subset K$ ist. Für beliebiges $x \in G$ gilt $G \subset x+K$, d.h. $\mathscr{G}$ enthält eine kompakte Menge und besitzt damit einen Adhärenzpunkt. Nach [5, § III.4], konvergiert jeder Cauchy-Filter, der einen Adhärenzpunkt besitzt. Der Raum $(E, \Lambda)$ ist also vollständig.

Satz 3. Der Quotientenraum $(E / H, \Lambda / H)$ (in der Kategorie der Limesräume) eines lokalkompakten Limesvektorraumes $(E, A)$ nach einem Unterraum $H$ ist lokalkompakt.

Beweis. Das $\Lambda$-Ideal $(\Lambda / H) 0$ wird von den Filtern $\varphi(\mathcal{F}), \mathcal{F} \in \Lambda 0$, erzeugt ( $\varphi: E \rightarrow E / H$ ist die natürliche Abbildung). Weil das stetige Bild einer kompakten Menge kompakt ist [5], ist der Quotientenraum lokalkompakt.

Wir wissen nicht, ob es unendlichdimensionale, topologische Vektorräume gibt, die $(m)$-Objekte in irgendeiner der Kategorien V'e, V/EC , $\mathcal{V} M$ sind. Es gilt aber den

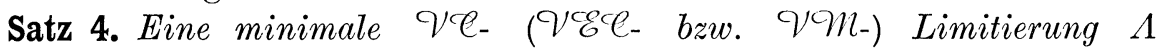
auf einem Vektorraum $E$ ist genau dann eine Topologie, wenn der Dualraum $E^{\prime}$ Punkte von $E$ separiert und $\Lambda$ ist dabei gleich der schwachen Topologie $\sigma\left(E, E^{\prime}\right)$ auf $E$.

Beweis. Nach [5, § III], ist eine Linearform auf $E$ genau dann $\Lambda$-stetig, wenn sie bezüglich der zu $\Lambda$ assoziierten, lokalkonvexen Topologie $\omega(\Lambda) \leq \Lambda$ stetig ist. Es ist folglich $\omega(\Lambda)$ separiert, falls $E^{\prime}$ Punkte von $E$ separiert. Die Limitierung $\Lambda$ ist somit gleich der gröbsten lokalkonvexen Topologie, die den Dualraum $E^{\prime}$ hat, d.h. $A=\sigma\left(E, E^{\prime}\right)$. Die Umkehrung ist trivial. 


\section{3. $\mathcal{V Q E}^{+}+$und $\mathcal{V}^{\circ} \mathcal{E C}^{\dagger}$-Limitierungen}

Nach einem Lemma in [6] ist (mit einer kleinen Verallgemeinerung) ein Filter $\mathcal{F}$ auf einem Vektorraum $E$ genau dann ausgeglichen, wenn er eine Basis aus kreisförmigen Mengen besitzt und $\lambda \mathcal{F} \leq \mathcal{F}$ für jedes $\lambda \neq 0, \lambda \in \mathbf{K}$ ist. Diese Charakterisierung benutzen wir im nächsten Beweis.

Lemma 5. Es sei $\left.(E, \Lambda) \in\right|^{\mathcal{V}^{\bar{C}} \dagger} \mid$. Für jedes $\mathcal{F} \in \Lambda 0$ gibt es einen stark ausgeglichenen Filter $\mathscr{G} \in \Lambda 0$ mit $\mathscr{G} \leq \mathcal{F}$, der eine abzählbare Basis besitzt.

Beweis. Für jedes $\mathcal{F} \in \Lambda 0$ gibt es einen stark ausgeglichenen Filter $\mathscr{C}^{\prime}$ und ferner einen Filter $\mathscr{C} \in \Lambda 0$ mit abzählbarer Basis $\left\{H_{n} \mid n \in \mathbf{N}\right\}$ derart, dass $x \leq \mathscr{G}^{\prime} \leq \mathcal{F}$ ist. Da $X \leq \mathscr{C}^{\prime}=\mathscr{G}^{\prime}+\mathscr{C}^{\prime}$ ist, kann eine Familie $\left(G_{n}^{(m)}\right)_{(m, n) \in \mathbf{N} \times \mathbf{N}}$ aus kreisförmigen Mengen $G_{n}^{(m)} \in \mathcal{C}^{\prime}$ durch Induktion so konstruiert werden, dass die Beziehungen $H_{n} \supset G_{n}^{(1)}$, $G_{n}^{(m)} \supset G_{n}^{(m+1)}+G_{n}^{(m+1)}$ und $G_{n}^{(m)} \supset G_{n+1}^{(m)}$ für alle $m$ und $n$ gelten. Sei $C_{g}$ der von der Filterbasis $\left\{(1 / k) G_{n}^{(m)} \mid(k, m, n) \in \mathbf{N} \times \mathbf{N} \times \mathbf{N}\right\}$ erzeugte Filter. Dieser ist ausgeglichen, weil für jedes $\lambda \neq 0, \lambda \in \mathbf{K}$, und jedes $(k, m, n) \in \mathbf{N} \times \mathbf{N} \times \mathbf{N}$ ein $l \in \mathbf{N}$ so gewählt werden kann, dass $\lambda(1 / k) G_{n}^{(m)}=|\lambda|(1 / k) G_{n}^{(m)} \supset(1 / k l) G_{n}^{(m)}$ gilt. Weiter ist nach der Konstruktion $\mathscr{G}=\mathscr{C}_{\mathrm{g}}+\mathscr{C}_{\mathrm{g}}$ und $\mathscr{\ell} \leq \mathscr{C}_{\mathrm{g}} \leq \mathscr{C}^{\prime}$, d.h. $\mathscr{G}$ hat die in Lemma 5 geforderten Eigenschaften.

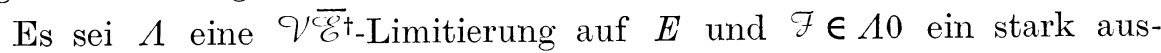
geglichener Filter mit einer abzählbaren Basis $\left\{F_{n} n \in \mathbf{N}\right\}$ aus kreisförmigen Mengen. Durch Induktion konstruiert man eine neue Basis $\left\{W_{n} \mid n \in \mathbf{N}\right\}$ aus kreisförmigen Mengen derart, dass $W_{n+1}+W_{n+1}+W_{n+1} \subset F_{n} \cap W_{n}$ für jedes $n \in \mathbf{N}$ ist. Durch

$$
\begin{aligned}
& \gamma(0)=0, \quad \gamma(x)=1 \text { falls } x \notin W_{1} \\
& \gamma(x)=1 / 2^{k} \text { falls } x \in W_{k} \text { und } x \notin W_{k+1} \\
& \delta_{F}(x, y)=\inf \sum_{i=1}^{p} \gamma\left(t_{i}-t_{i-1}\right),
\end{aligned}
$$

wo das Infimum über alle endlichen Folgen $\left(t_{i}\right)_{0 \leqq i \leqq p}$ mit $t_{0}=x$ und $t_{p}=y$ genommen wird, wird eine Abbildung $\delta_{\bar{F}}: E \times E \rightarrow \mathbf{R}$ definiert. Genau wie für topologische Vektorräume (siehe [7] und [9]) zeigt man, dass $\delta_{F}$ eine translationsinvariante Metrik ist, dass die Abbildung $\varrho_{\bar{F}}: E \rightarrow \mathbf{R}, \varrho_{\bar{F}}(x)=\delta_{\bar{F}}(x, 0)$, die Bedingung

(iv) aus $|\lambda| \leq 1$ folgt $\varrho(\lambda x) \leq \varrho(x)$

erfüllt und dass die Filterbasis $\left\{B_{\varepsilon}\left(\delta_{\bar{F}}\right) \mid \varepsilon>0\right\}$ den Filter $\mathcal{F}$ wieder erzeugt. Dabei bezeichnet $B_{\varepsilon}\left(\delta_{\bar{F}}\right)$ die Menge $\left\{x \in E \mid \delta_{\bar{F}}(x, 0) \leq \varepsilon\right\}$. Wenn 
$\mathcal{I}$ die Menge der stark ausgeglichenen Filter in $\Lambda 0$ durchläuft, erhalten wir eine Familie $\left(\delta_{F}\right)$ von translationsinvarianten Metriken, die die Limitierung $\Lambda$ wieder definieren. Da alle $\delta_{F}$ translationsinvariant sind, hat jedes $\varrho_{F}$ die Eigenschaften:

(i) $\varrho(x)=0$ genau dann, wenn $x=0$ ist

(ii) $\varrho(x)=\varrho(-x)$

(iii) $\varrho(x+y) \leq \varrho(x)+\varrho(y)$.

Sei $x \in E$ beliebig. Da $V x \in \Lambda 0$ ist, gibt es ein stark ausgeglichenes $\mathcal{F} \in \Lambda 0$ mit $\boldsymbol{V} x \geq \mathcal{F}$. Der Filter $\varrho_{F}(\boldsymbol{V} x)$ ist gegen Null konvergent.

Die Menge der translationsinvarianten Metriken auf $E$ ist durch die Relation $" \delta_{1}$ definiert einen gröberen Filter als $\delta_{2}{ }^{\prime}$, die wir mit $\delta_{1} \leq \delta_{2}$ bezeichnen, vorgeordnet. Es ist $\delta_{1} \leq \delta_{2}$ genau dann, wenn für jedes $\varepsilon>0$ ein $\eta>0$ existiert, so dass für jedes $x \in E$ aus $\delta_{2}(x, 0) \leq \eta$ folgt $\delta_{1}(x, 0) \leq \varepsilon$. Für je zwei stark ausgeglichene $\mathcal{F}$ und $\varphi_{\mathcal{f}}$ in $\Lambda 0$ gibt es ein stark ausgeglichenes $\mathcal{C}$ in $\Lambda 0$, so dass $\delta_{\vec{F}} \geq \delta_{\mathcal{\gamma}}$ und $\delta_{\mathcal{G}} \geq \delta_{\mathcal{H}}$ ist.

Wir fassen zusammen:

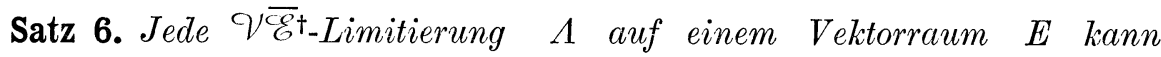
mit Hilfe einer durch die Relation $\geq$ gerichteten Menge 4 translationsinvarianter Metriken derart definiert werden, dass die Abbildungen $\varrho: x \mapsto$ $\delta(x, 0), \delta \in \Delta$, (iv) genügen und die Bedingung

(v) für jedes $x \in E$ gibt es ein $\delta \in \Delta$, so dass der Filter $\delta(\boldsymbol{V} x, 0)$ gegen Null konvergiert

erfüllt ist. Jede durch $\geq$ gerichtete Menge $\Delta$ translationsinvarianter Metriken, die (v) genügt und für die für jedes $\delta \in \Delta$ die assoziierte Abbildung @

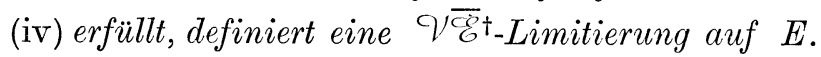

Beweis für die letzte Behauptung: Wir setzen $\Lambda 0=\left\{C_{1} \in \mathbf{F}(E) \mid C_{1} \geq g_{\delta}\right.$, $\delta \in \Delta\}$ und $A x=x+\Lambda 0$, wobei $\mathcal{F}_{\delta}$ den von der Filterbasis $\left\{B_{\varepsilon}(\delta) \mid \varepsilon>0\right\}$ erzeugten Filter bezeichnet. Da $\Delta$ durch $\geq$ gerichtet ist, ist $\Lambda$ eine Limitierung. Es ist $\lambda_{\mathcal{F}}=\mathcal{F}_{\delta}(\delta \in \Delta)$ für jedes $\lambda \neq 0$ und wegen (iv) besitzt $\mathcal{F}_{\delta}$ eine Basis aus kreisförmigen Mengen. Nach dem im Anfang dieser Nummer erwähnten Lemma in [6] ist der Filter $\mathcal{F}_{\delta}$ ausgeglichen. Er ist sogar stark ausgeglichen, denn für jedes $\varepsilon>0$ ist $B_{(1 / 2) \varepsilon}(\delta) \subset B_{(1 / 2) \varepsilon}(\delta)+B_{(1 / 2) \varepsilon}(\delta) \subset B_{\varepsilon}(\delta)$, d.h. $\mathcal{F}_{\delta}=\mathcal{F}_{\delta}+\mathcal{F}_{\delta}$. Da 40 von stark ausgeglichenen Filtern erzeugt wird und $V x$ für jedes $x \in E$ wegen (v) in $\Lambda 0$ ist, ist $\Lambda$ eine Vektorraumlimitierung (vgl. [5, § III.5]).

Kor. 6. Jede $\sqrt{\bar{C}} \bar{C}_{-L i m i t i e r u n g}$ auf $E$ ist induktiver Limes (in der Kategorie der Limesräume) metrisierbarer Topologien auf $E$.

Bemerkung. Jede $\mathcal{V} \overline{\mathscr{E}}$-Limitierung $\Lambda$ auf $E$ ist induktiver Limes 
einer Familie von Topologien auf $E$, denn für jedes stark ausgeglichenes $\mathcal{F} \in \Lambda 0$ ist, wie man leicht nachprüft, für jedes $x \in E$ der Filter $x+\mathcal{F}$ Umgebungsfilter von $x$ in bezug auf eine Topologie auf $E$.

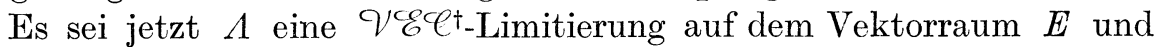
$\psi$ der von $A$ definierte, saturierte Filter auf der Menge $Q(E)$ der Pseudonormen auf $E$ (siehe [2]). Für beliebiges $\mathcal{F} \in \Lambda 0$ gibt es ein ausgeglichenes $\mathscr{G}^{\prime}$ mit einer Basis aus absolutkonvexen Mengen und ein $x \in \Lambda 0$ mit einer abzählbaren Basis $\left\{H_{n} \mid n \in \mathbf{N}\right\}$, so dass $\mathcal{X} \leq \mathscr{C}^{\prime} \leq \mp$ ist. Für jedes $n \in \mathbf{N}$ gibt es ein $G_{n} \in \varrho_{y}^{\prime}$ mit $H_{n} \supset G_{n}$. Für den von der Filterbasis $\left\{(1 / k) G_{n} \mid(k, n) \in \mathbf{N} \times \mathbf{N}\right\}$ erzeugten Filter $C_{\text {gilt: }} \mathscr{X} \leq C_{1} \leq \mathscr{C}_{1}^{\prime}$. Es gibt somit eine Menge $\Phi$ aus ausgeglichenen Filtern mit abzählbaren Basen aus absolutkonvexen Mengen, so dass $\mathcal{F}$ genau dann in 10 ist, wenn ein $\mathscr{C}_{\mathcal{G}} \in \Phi$ mit $\mathscr{C}_{\mathcal{F}} \leq \mathcal{F}$ existiert. Die Mengen $\left\{g_{G} \mid G \in \mathscr{G}_{\mathcal{G}} G\right.$ absolutkonvex $\}$,

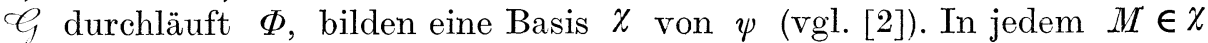
gibt es eine aufsteigende unendliche Folge $q_{1}^{(M)} \leq q_{2}^{(M)} \leq \ldots$ von Pseudonormen derart, dass für jedes $q \in M$ ein $\lambda>0$ und ein $n \in \mathbf{N}$ mit $q \leq \lambda q_{n}^{(M)}$ existieren. Durch

$$
\varrho_{M}(x)=\sum_{n=1}^{\infty} \frac{1}{2^{n}} \frac{q_{n}^{(M)}(x)}{1+q_{n}^{(M)}(x)}
$$

wird eine Abbildung $\varrho_{M}: E \rightarrow \mathbf{R}$ definiert (wir setzen $\xi /(1+\xi)=1$ für $\xi=\infty)$. Genau wie in der Theorie der lokalkonvexen Räume zeigt man (für die Einzelheiten verweisen wir auf [7] und [9]), dass $\varrho_{M}$ die Eigenschaften (i) bis (iv) hat und dass die Mengen $\left\{x \in E\left\lfloor\varrho_{M}(x) \leq \varepsilon\right\}, \varepsilon>0\right.$, den von $M$ definierten Filter erzeugen. In dieser Weise erhalten wir eine Menge $\Delta=\left\{\delta_{M} \mid M \in \chi\right\}$ translationsinvarianter Metriken $\delta_{M}:(x, y) \mapsto$ $\varrho_{M}(x-y)$, die die Limitierung $\Lambda$ definieren.

Satz 7. Es sei $\Lambda$ eine $\sqrt{\bar{C}}$ t-Limitierung auf $E, \Delta$ eine Menge translationsinvarianter Metriken, die die Limitierung $\Lambda$ definieren und $P$ die Menge der Abbildungen $\varrho: x \mapsto \delta(x, 0), \delta \in \Delta$. Dann wird die Quotientenlimitierung (in der Kategorie der Limesräume) auf einem Quotientenraum $E / H$ nach einem abgeschlossenen Unterraum $H$ von $E$ von den translationsinvarianten Metriken $\hat{\delta}:(x, y) \rightarrow \underline{\varrho}(\hat{x}-\hat{y}), \varrho \in P$, definiert. Dabei ist $\hat{\varrho} \quad$ durch

$$
\hat{\varrho}(\hat{x})=\inf _{y \in \hat{x}} \varrho(y), \quad \hat{x}=\varphi(x)
$$

$(\varphi: E \rightarrow E / H$ ist die natürliche Abbildung) definiert, und für jedes $\varrho \in P$ hat $\hat{\varrho}$ die Eigenschaften (i) bis (iv) und die Menge der Metriken $\hat{\delta}$ die Eigenschaft (v).

Beweis. Man verifiziert wie in der Theorie der metrisierbaren, topologischen Vektorräume, dass die Abbildung $\varrho \varrho$ die Eigenschaften (i) bis (iv) 
hat. Die Abbildungen $\hat{\delta}:(\hat{x}, \hat{y}) \mapsto \hat{\varrho}(\hat{x}-\hat{y}), \varrho \in P$, sind also translationsinvariante Metriken. Sie definieren die Quotientenlimitierung $A / H$ auf $E / H$, denn für jedes $\varepsilon>0$ ist $\left.\varphi\left(B_{\varepsilon}(\delta)\right) \subset B_{\varepsilon}(\hat{\delta})\right) \subset \varphi\left(B_{2 \varepsilon}(\delta)\right)$. Das $\wedge$-Ideal $(\Lambda / H) 0$ wird ja gerade von den Filtern $\varphi\left(\left[\left\{B_{\varepsilon}(\delta) \mid \varepsilon>0\right\}\right]\right), \delta \in \Delta$, erzeugt. Die Bedingung (v) ist automatisch erfüllt.

Satz 8. Der Quotientenraum $(E / H, \Lambda / H)$ nach einem abgeschlossenen Unterraum $H$ eines vollständigen Raumes $(E, \Lambda) \in||^{\mid \bar{C}} \dagger \mid$ ist vollständig.

Beweis. Sei $\mathscr{C}$ ein Cauchy-Filter in dem Quotientenraum. Da $\Lambda / H$ der Abzählbarkeitsbedingung $(\dagger)$ genügt, gibt es eine Cauchy-Folge $\left(\hat{x}_{n}\right)_{n \in \mathrm{N}}$ in $(E / H, \Lambda / H)$, deren assoziierter Elementarfilter feiner als $\mathscr{G}$ ist. Sei $\Delta$ eine Menge translationsinvarianter Metriken, die $\Lambda$ definieren. Dann gibt es ein $\delta \in \Delta$ derart, dass die Folge $\left(\hat{x}_{n}\right)_{n \in \mathbb{N}}$ im metrischen Raum $(E / H, \hat{\delta})(\hat{\delta}$ ist wie in Satz 7 definiert) eine Cauchy-Folge ist. Genau wie für Banach-Räume zeigt man, dass $\left(\hat{x}_{n}\right)_{n \in \mathbf{N}}$ eine bezüglich $\hat{\delta}$ und damit auch bezüglich $\Lambda / H$ konvergente Teilfolge besitzt (wir verweisen auf [9]). Der Cauchy-Filter $\varphi$, hat einen Adhärenzpunkt und ist somit konvergent $[5, \S \mathrm{III}]$.

Ein Limesraum $(E, \lambda)$ heisst regulär $[\tilde{5}]$, wenn mit einem Filter $\mathcal{F}$ auch der von den Hüllen $\bar{F}(F \in \mathcal{F})$ erzeugte Filter $\mathcal{F}$ gegen $x \in E$

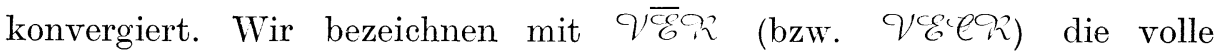
Unterkategorie von $\mathcal{V} \mathscr{L}$, die genau alle reguläre Objekte in $\overline{\mathcal{Y}}$ (bzw. Vece) enthält.

Satz 9. Jeder vollständige, metrisierbare, topologische Vektorraum ist (m)-Objekt in VGogt*.

Beweis. Es sei $\left(E, \tau_{0}\right)$ ein vollständiger, metrisierbarer, topologischer Vektorraum, $\Lambda$ eine Limitierung auf $E$ mit $\Lambda \leq \tau_{0}$ und $(E, \Lambda) \in|\sqrt{\mathcal{E}} \mathcal{L}+*|$ und schliesslich sei $\Delta$ eine Menge translationsinvarianter Metriken, die $\Lambda$ definieren. Jedes $\delta \in \Delta$, das einen gröberen Filter als der Nullumgebungsfilter in $\left(E, \tau_{0}\right)$ definiert, definiert eine Vektorraumtopologie auf $E$. Der Filter $\delta(\boldsymbol{V} x, 0)$ ist ja für jedes $x \in E$ gegen Null konvergent. Die Limitierung $\Lambda$ ist also induktiver Limes der durch $\geq$ gerichteten Menge $\tau$ aller metrisierbaren Vektorraumtopologien $\tau$ auf $E$ mit $\tau_{0} \geq \tau \geq \Lambda$. Für jedes $\tau \in \widetilde{\tau}$ sei $\left(\widetilde{E}_{\tau}, \tilde{\tau}\right)$ die vollständige Hülle von $(E, \tau)$ und für je zwei $\tau$ und $\tau^{\prime}$ in $\widetilde{\tau}$ mit $\tau \geq \tau^{\prime}$ sei $h_{\tau^{\prime} \tau}:\left(\widetilde{E}_{\tau}^{\prime}, \tilde{\tau}\right) \rightarrow\left(\widetilde{E}_{\tau^{\prime}}, \tilde{\tau}^{\prime}\right)$ die stetige Fortsetzung der Inklusion $(E, \tau) \rightarrow\left(\widetilde{E}_{\tau^{\prime}}^{\prime}, \tilde{\tau}^{\prime}\right)$. Es ist $h_{\tau^{\prime \prime} \tau^{\prime}} \circ h_{\tau^{\prime} \tau}=h_{\tau^{\prime \prime} \tau}$ für $\tau \geq \tau^{\prime} \geq \tau^{\prime \prime}$.

Weil $(E, \Lambda)$ regulär ist, besitzt die Identität $(E, \tau) \rightarrow(E, \Lambda)$ für jedes $\tau \in \widetilde{G}$ eine stetige lineare Fortsetzung $h_{\tau}:\left(\tilde{E}_{\tau}^{\prime}, \tilde{\tau}\right) \rightarrow(E, \Lambda)$ (vgl. [12]). Für jedes $z \in E$ sei $h_{\tau}(z)$ der Konvergenzpunkt bezüglich $\Lambda$ eines Cauchy-Filters $\mathcal{J}$ in $(E, \tau)$, der in $\left(\tilde{E}_{t}, \tilde{\tau}\right)$ einen gegen $z$ konvergierenden Filter erzeugt. Um die Stetigkeit der linearen Abbildung $h_{\tau}$ nachzu- 
weisen, betrachten wir den Nullumgebungsfilter $\gamma_{\tau}$ in $(E, \tau)$ und den von den abgeschlossenen Hüllen $\bar{V}^{\tilde{\tau}}\left(V \in \mathcal{V}_{\tau}\right)$ in $\left(\widetilde{E}_{\tau}, \tilde{\tau}\right)$ erzeugten Nullumgebungsfilter $\mathcal{V}_{\tilde{\tau}}$ in $\left(\tilde{E}_{\tau}, \tilde{\tau}\right)$ (vgl. [9]). Aus $z \in \bar{V}^{\tilde{\tau}}$ und $V \in \mathscr{C}_{1} \geq z+\mathcal{V}_{\tilde{\tau}}$ folgt $h_{\tau}(z)=\lim _{\Lambda} C_{E} \in \bar{V}^{\Lambda}$, wo $\bar{V}^{\Lambda}$ die Hülle von $V$ bezüglich $\Lambda$ und $\mathscr{C}_{E}$ der Spur von $\mathscr{C}_{\mathcal{J}}$ auf $E$ bezeichnen. Es ist also $h_{\tau}\left(\bar{V}^{\tilde{\tau}}\right) \subset \bar{V}^{\Lambda}$ für jedes $V \in \mathcal{V}_{\tau}$, d.h. $h_{\tau}\left(\gamma_{\tau}\right) \geq{\overline{V^{\prime}}}_{\tau}^{\Lambda}$. Da $\Lambda$ regulär ist, ist $\bar{\vartheta}_{\tau}^{A} \in \Lambda 0$ und $h_{\tau}$ ist somit stetig. Offensichtlich ist $h_{\tau^{\prime}}=h_{\tau^{\prime} \tau} \circ h_{\tau}$ für je zwei $\tau$ und $\tau^{\prime}$ in $\tau$ mit $\tau \geq \tau^{\prime}$.

Für jedes $\tau \in \tau$ sei $k_{\tau}:(E, \tau) \rightarrow\left(\tilde{E}_{\tau}, \tilde{\tau}\right)$ die Inklusion. Wir betrachten den induktiven Limes $(E$, ind $\tilde{\tau}$ ) (in der Kategorie der Limesvektorräume) der Räume $\left(\tilde{E}_{\tau}, \tilde{\tau}\right), \tau \in \mathcal{F}$, bezüglich der Abbildungen $h_{\tau^{\prime} \tau}, \tau \geq \tau^{\prime}$. Für jeden Filter $\mathcal{F} \in \Lambda 0$ gibt es ein $\tau \in \mathcal{F}$, so dass $k_{\tau}(\mathcal{F})$ bezüglich $\tilde{\tau}$ gegen Null konvergiert. Da das $\wedge$-Ideal (ind $\tilde{\tau}$ ) 0 von den Filtern $h_{\tau}\left(\mathcal{V}_{\tau}\right), \tau \in \mathcal{\tau}$, erzeugt wird, ist folglich ind $\tilde{\tau} \leq \Lambda$. Die Limitierung ind $\tilde{\tau}$ ist aber die feinste Vektorraumlimitierung auf $E$, für die alle $h_{\tau}$ stetig sind. Sie ist also mit $A$ identisch. Im kommutativen Diagramm $(\tau \in \tau)$

$$
\begin{array}{rll}
k_{\tau}^{\prime} \nearrow & (\tilde{E}, \tilde{\tau}) & \\
\left(E, \tau_{0}\right) & \downarrow_{\tau} & \left(\tilde{E}_{\tau}^{\prime} / K_{\tau}, \tilde{\tau} / K_{\tau}\right) \\
i d_{E} \searrow & { }^{\prime} & \swarrow h_{\tau}^{\prime}
\end{array}
$$

ist das rechte Dreieck die kanonische Zerlegung von $h_{\tau}$ in einen Epimorphismus $\varphi$ und einen anschliessenden Monomorphismus $h_{\tau}^{\prime}$, und $k_{\tau}^{\prime}$ ist die natürliche Abbildung. Die Limitierung $\Lambda=$ ind $\tilde{\tau}$ ist offensichtlich die feinste Vektorraumlimitierung auf $E$, für welche alle $h_{\tau}^{\prime}$ stetig sind. Da der Quotientenraum $\left(\tilde{E}_{\tau} / K_{\tau}, \tilde{\tau} / K_{\tau}\right)$ ein vollständiger, metrisierbarer, topologischer Vektorraum und $\varphi \circ k_{\tau}^{\prime}$ ein Bimorphismus ist, ist diese Abbildung ein Isomorphismus. Es folgt, dass die Limitierung $\Lambda$ mit $\tau_{0}$ identisch sein muss. Satz 9 ist damit bewiesen.

Kor. 9.1. Jeder Bimorphismus $f:(E, \tau) \rightarrow\left(F, A^{\prime}\right)$ von einem vollständigen, metrisierbaren, topologischen Vektorraum $(E, \tau)$ auf einen Raum $\left(F, \Lambda^{\prime}\right) \in\left|\mathcal{V} \bar{E} R^{*} *\right|$ ist ein Isomorphismus.

Kor. 9.2. Jeder (F)-Raum ist $(m)$-Objekt in qugeont*.

\section{Der Graphensatz}

Es sei $\mathcal{K}$ gleich einer der Kategorien $\vee \mathscr{L}, \mathcal{Y E}, \sqrt{\mathscr{E}}, \mathcal{V}$, vege, vom.

Satz 10. Eine lineare Abbildung $f:(E, A) \rightarrow\left(F, A^{\prime}\right)$ von einem Raum 
$(E, \Lambda) \in|\mathcal{K}| \quad\left(\left|\mathcal{K}^{\dagger}\right|,\left|\mathcal{K}^{l c}\right|,\left|\mathcal{K}^{+l c}\right|,\left|\mathcal{V}^{\bar{C}}+*\right|,\left|\mathcal{V E C E}^{+*}\right|\right.$ bzw. $\left.|\mathcal{V} M+*|\right)$ nach einem $(m)$-Objekt $\left(F, \Lambda^{\prime}\right)$ in derselben Kategorie ist genau dann stetig, wenn der Graph $G_{f}$ von $f$ im Produktraum $E \times F$ abgeschlossen ist.

Beweis. Ist $f$ stetig, so ist $G_{f}$ abgeschlossen (siehe [1]). Ist umgekehrt $G_{f}$ abgeschlossen, so ist auch der Graph $G_{-f}$ der Abbildung $x \mapsto-f(x)$ abgeschlossen. Die Abbildungen $k: E \rightarrow\left(E \times F^{\prime}\right) / G_{-f}$ und $l: F \rightarrow(E \times F) / G_{-f}$ seien die Kompositionen der natürlichen Abbildungen $E \rightarrow E \times F \rightarrow$ $\left(E \times F^{\prime}\right) / G_{-f}$ bzw. $F \rightarrow E \times F \rightarrow(E \times F) / G_{-f}$. Diese sind $\left(\Lambda,\left(\Lambda \times \Lambda^{\prime}\right) / G_{-f}\right)-$ bzw. $\left(\Lambda^{\prime},\left(\Lambda \times \Lambda^{\prime}\right) / G_{-f}\right)$-stetig. Die Limitierung $\left(\Lambda \times \Lambda^{\prime}\right) / G_{-f}$ ist separiert, weil $G_{-f}$ abgeschlossen ist [14]. Ferner ist sie eine Marinescu-, (stark) ausgeglichene oder absolutkonvexe Limitierung oder sie erfüllt $(\dagger)$ oder $(l c)$, falls dies für $\Lambda$ und $\Lambda^{\prime}$ gilt ([6], [8], [13], [2], Satz 3). Der Quotientenraum eines (stark) ausgeglichenen Raumes ist (stark) ausgeglichen, weil das lineare Bild eines (stark) ausgeglichenen Filters (stark) ausgeglichen ist. Schliesslich ist nach [4] und Satz 8 der Raum $\left(\left(E \times F^{\prime}\right) / G_{-f},\left(\Lambda \times \Lambda^{\prime}\right) / G_{-f}\right)$

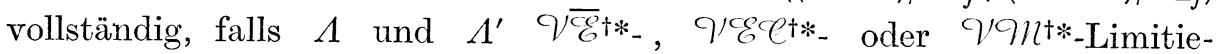
rungen sind. Da $\left(F, A^{\prime}\right)$ ein $(m)$-Objekt und - wie man leicht nachprüft die Abbildung $l$ bijektiv ist, ist $l^{-1}$ und damit auch $f=l^{-1} \circ l$ stetig.

\section{Vollständigkeit und K-Einbettungsvollständigkeit}

Es sei $\mathcal{K}$ eine volle Unterkategorie von $\mathcal{V} \mathscr{L}$.

Definition. Ein Raum $(E, A) \in|\mathcal{K}|$ heisse K-einbettungsvollständig, wenn für jede Einbettung $i:(E, \Lambda) \rightarrow\left(F, \Lambda^{\prime}\right)$ von $(E, \Lambda)$ in einen Raum $\left(F, \Lambda^{\prime}\right) \in|\mathcal{K}|$ das Bild $i(E)$ von $E$ ein abgeschlossener Unterraum von $\left(F, \Lambda^{\prime}\right)$ ist.

Satz 11. Jeder vollständige Raum $(E, \Lambda) \in|\mathcal{K}|$ ist K-einbettungsvollständig.

Beweis. Sei $i:(E, \Lambda) \rightarrow\left(F, \Lambda^{\prime}\right)\left(\left(F^{\prime}, \Lambda^{\prime}\right) \in|\mathcal{K}|\right)$ eine Einbettung und $y \in F$ ein beliebiger an $i(E)$ adhärenter Punkt. Sei ferner $\mathscr{G} \in \Lambda^{\prime} y$ ein Filter der $i(E)$ als Element enthält. Das Urbild $i^{-1}\left(\mathscr{G}_{i(E)}\right)$ der Spur von $\mathscr{C}_{\text {auf }} i(E)$ ist ein Cauchy-Filter in $(E, A)$, weil $\mathscr{C}_{i(E)}$ im Unterraum $i(E)$ ein Cauchy-Filter ist. Sei $x$ der Konvergenzpunkt von $i^{-1}\left(\mathscr{C}_{i(E)}\right)$. Dann ist $y=i(x) \in i(E)$. Der Unterraum $i(E)$ ist somit abgeschlossen für jede Einbettung $i$, d.h. $(E, \Lambda)$ ist K-einbettungsvollständig.

Sei $\mathcal{F}$ ein Filter auf einem Velktorraum $E$. Mit $\mathbf{K} \cdot \mathcal{F}$ bezeichnen wir den von den Mengen $\mathbf{K} \cdot F=\{\lambda x \mid \lambda \in \mathbf{K}, x \in F\}, F \in \mathcal{F}$, erzeugten Filter auf E. Mit Ultra-Cauchy-Filter verstehen wir einen Cauchy-Filter, der ein Ultrafilter ist.

Satz 12. Es sei $\mathcal{K}$ gleich einer der Kategorien Ve, qege oder

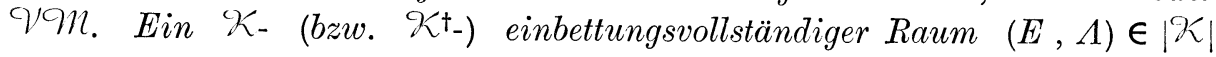


(bzw. $\mathcal{K}^{\dagger} \mid$ ) ist genau dann vollständig, wenn es für jeden Ultra-CauchyFilter $\mathcal{U}$ in $(E, \Lambda)$ ein $\mathcal{F} \in \Lambda 0$ gibt, so dass $\mathcal{U} \geq \mathbf{K} \cdot \mathcal{F}$ ist.

Beweis. a) Falls der Raum $(E, A)$ vollständig ist, so ist er auch einbettungsvollständig nach Satz 11. Sei $U$ ein Ultra-Cauchy-Filter in $(E, A)$. Dann gibt es wegen der Vollständigkeit ein $\mathcal{F} \in \Lambda 0$ mit absolutkonvexer Basis und ein $x \in E$, so dass $\mathcal{U} \geq x+\mathcal{F} \geq \mathbf{K} \cdot(\boldsymbol{V} x+\mathcal{F})$ ist.

b) Sei jetzt $(E, \Lambda)$ ein $\mathcal{K}_{-}$(bzw. $\mathcal{K}^{\dagger}$ ) einbettungsvollständiger Raum mit der in Satz 9 erwähnten Eigenschaft. Wir nehmen an, dass es einen nicht-konvergenten Ultra-Cauchy-Filter $\mathcal{U}$ in $(E, \Lambda)$ gibt und zeigen, dass diese Annahme zu einem Widerspruch führt. Es sei $\Omega$ ein Vektorraum, der $E$ als echten Unterraum enthält. Ferner seien $z \in \Omega \backslash E$ ein beliebiger Punkt, $E_{1}$ die lineare Hülle von $E \cup\{z\}$ in $\Omega$ und $\psi$ der von $\Lambda$ definierte, saturierte Filter auf $Q(E)$. Für jede Pseudonorm $q$ auf $E$ ist die Abbildung $q_{1}: E_{1} \rightarrow \mathbf{R}^{+} \cup\{\infty\}$,

$$
q_{1}(x+\lambda z)=\lim q(x+\lambda \mathcal{U}), \quad x \in E, \quad \lambda \in \mathbf{K},
$$

eine Pseudonorm auf $E_{1}$ und eine Fortsetzung von $q$ (wir betrachten $\mathbf{R}^{+} \cup\{\infty\}$ als Ein-Punkt-Kompaktifikation von $\mathbf{R}^{+}$). Sei für jedes $M \subset Q(E)$ die Menge $M_{1}$ durch $M_{1}=\left\{p \in Q\left(E_{1}\right) \mid \exists q \in M\right.$ mit $\left.p \leq q_{1}\right\}$ definiert. Ist dabei $M$ saturiert, so ist es auch $M_{1}$.

Wir zeigen, dass der Filter $\psi_{1}=\left[\left\{M_{1} \mid M \in \psi\right\}\right]$ auf $Q\left(E_{1}\right)$ eine absolutkonvexe Limitierung auf $E_{1}$ definiert. Offensichtlich ist $\lambda \psi_{1}=\psi_{1}$ für jedes $\lambda>0$. Sei nun $x+\lambda z \in E_{1}(x \in E, \lambda \in \mathbf{K})$ beliebig. Weil $2 l^{\prime}=$ $x+\lambda \varkappa$ ein Ultra-Cauchy-Filter ist, gibt es eine Menge $M \in \psi$ derart, dass $2 l^{\prime}-\imath^{\prime} \geq \mathcal{F}_{M}$ und $\imath^{\prime} \geq \mathbf{K} \cdot \mathcal{F}_{M}$ ist. Für jedes $q \in M$ existiert dann eine Menge $U \in \imath^{\prime}$, so dass $q(v)<\infty$ und $q(u-v) \leq 1$ für jedes Paar $(u, v) \in U \times U$ ist. Man folgert hieraus, dass $q(U) \subset[0,1+q(v)]$ für beliebiges festes $v \in U$ und ferner, dass $q_{1}(x+\lambda z)=\lim q\left(2 l^{\prime}\right)<\infty$ für jedes $q \in M$ ist. Der Filter $\psi_{1}$ definiert somit eine absolutkonvexe Limitierung $\Lambda_{1}$ auf $E_{1}$. Es ist klar, dass die Inklusion $i:(E, \Lambda) \rightarrow\left(E_{1}, \Lambda_{1}\right)$ eine Einbettung ist ([2]).

Offensichtlich ist $\left(E_{1}, \Lambda_{1}\right)$ ausgeglichen (bzw. erfüllt $\left(E_{1}, \Lambda_{1}\right)$ die Bedingung $(\dagger))$, wenn dies für $(E, \Lambda)$ gilt. Ist $\Lambda$ eine Marinescu-Limitierung, so besitzt $\psi$ eine Basis $\chi$ aus saturierten, ausgeglichenen Mengen derart, dass $q_{M} \in M$ für alle $M \in \chi$ ist [3, Satz 12]. Dabei ist die Pseudonorm $q_{M}$ durch

$$
q_{M}(x)=\left\{\begin{array}{l}
0 \text { falls } q(x)<\infty \text { für jedes } q \in M \text { ist } \\
\infty \text { falls es ein } q \in M \text { mit } q(x)=\infty \text { gibt }
\end{array}\right.
$$

definiert. Ist nun $q_{1}(x+\lambda z)=\lim q(x+\lambda \imath \iota) \infty$ für jedes $q \in M$, so gibt es für jedes solche $q$ ein $U \in \mathcal{U}$, so dass die Menge $q(x+\lambda U)$ beschränkt ist. Es ist also $\left(q_{M}\right)_{1}(x+\lambda z)=\lim q_{M}(x+\lambda \mathcal{U})=0$. Man 
erhält: $\left(q_{M}\right)_{1}=q_{M_{1}}$. Die Limitierung $\Lambda_{1}$ ist eine Marinescu-Limitierung nach [3].

Die Limitierung $\Lambda_{1}$ ist auch separiert. Seien $x+\lambda z \neq 0 \quad(x \in E$, $\lambda \neq 0)$ und $M \in \psi$ beliebig gewählt. Wir setzen wieder $\varkappa^{\prime}=x+\lambda^{2} \ell$. Wäre nun $\lim q\left(\iota^{\prime}\right) \leq 1$ für alle $q \in M$, so gälte $[0,1] \in q\left(\tau \ell^{\prime}\right)$ für jedes $q \in M$, und $2 \ell^{\prime}$ wäre dann feiner als $\mathcal{F}_{M}$ und somit gegen Null konvergent. Dies widerspricht aber unserer Annahme, dass $\mathcal{U}$ nicht konvergiert. Es gibt folglich ein $q$ in jedem $M \in \psi$, so dass $q_{1}(x+\lambda z)>1$ ist. Die Limitierung $\Lambda_{1}$ genügt $T_{1}$ und damit auch $T_{2}$ (siehe [13]).

Sei wieder $M \in \psi$ so gewählt, dass $\chi-{ } \ell \geq \mathcal{F}_{M}$ ist. Für jedes $q \in M$ gibt es ein $U \in \mathcal{U}$, so dass $[0,1] \cap q\left(x-U^{\prime}\right) \neq \phi$ für jedes $x \in U$ und jedes $U^{\prime} \in \mathcal{U}$ ist, oder anders ausgedrückt: so dass $q_{1}(x-z)=$ $\lim q(x-\mathcal{U}) \leq 1$ für jedes $x \in U$ ist. Es ist also $i(\mathcal{C}) \geq z+\mathcal{F}_{M_{1}}$. Der Unterraum $E$ ist somit dicht im Raum $\left(E_{1}, A_{1}\right)$, was der $\mathcal{K}$ - (bzw. $\left.K^{\dagger}-\right)$ Einbettungsvollständigkeit von $(E, A)$ widerspricht. Satz 12 ist damit bewiesen.

Kor. 12. Falls die Limitierung $A$ in Satz 12 gröber als eine Vektorraumtopologie ist, so ist sie genau dann vollständig, wenn sie K- (bzw. K' K-) einbettungsvollständig ist.

Beweis. In diesem Fall gibt es einen Filter $\mathcal{F} \in \Lambda 0$ aus absorbierenden Mengen. Die Bedingung in Satz 12 ist somit erfüllt.

\author{
Åbo Akademi \\ Mathematisches Institut \\ SF-20500 Åbo 50 \\ Finnland
}




\section{Literatur}

[1] BJon, S.: Beiträge zur Theorie der Limesräume. - Acta Acad. Aboensis, Ser. B $29, \mathrm{nr} 5(1969)$.

[2] -»- Über absolutkonvexe Limesvektorräume. - Soc. Sci. Fenn. Comment. Phys.-Math. 43, 181-188 (1973).

[3] -»- Die projektive, absolutkonvexe Tensorproduktlimitierung. - Ann. Acad. Sci. Fenn. Ser. A I 593 (1975).

[4] Cook, C. H., und Fischer, H. R.: Uniform Convergence Struktures. - Math. Ann. 173, 290-306 (1967).

[5] Fischer, H. R.: Limesräume. - Math. Ann. 137, 269-303 (1959).

[6] Frölicher, A., und Bucher, W.: Calculus in Vector Spaces without Norm. Lecture Notes in Math. 30 (1966).

[7] Horváth, J.: Topological Vector Spaces and Distributions. - Addison-Wesley Publ. Comp. 1966.

[8] JARchow, H.: Marinescu-Räume. - Comment. Math. Helv. 44, 138-163(1969).

[9] Kӧтне, G.: Topologische lineare Räume I. - Springer-Verlag 1960.

[10] Kutzler, K.: Eine Bemerkung über endlichdimensionale, separierte, limitierte Vektorräume. - Arch. der Math. 20, 165-168 (1969).

[11] -»- Bemerkungen über unendlichdimensionale, separierte Limesvektorräume und Limesgruppen. - J. reine angew. Math. 253, 98-116 (1972).

[12] ReEd, E. E.: Completions of Uniform Convergence Spaces. - Math. Ann. 194, 83-108 (1971).

[13] Wingren, C.-E.: Locally convex limit spaces. - Acta Acad. Aboensis, Ser. B 33, nr 5 (1973).

[14] Wloka, J.: Limesräume und Distributionen. - Math. Ann. 152, 351-409 (1963). 